\title{
New Traveling-Wave Antenna Resonating at 6 GHz Based on Artificial Transmission Line Metamaterial Structures for RF Portable Devices
}

\author{
Mohammad Alibakhshi Kenari \\ Electrical Engineering Department, Shahid Bahonar University of Kerman, Kerman, Iran. \\ Email: Naeem.alibakhshi@yahoo.com \\ Received May $6^{\text {th }}, 2013$; revised June $19^{\text {th }}, 2013$; accepted June $29^{\text {th }}, 2013$ \\ Copyright (C) 2013 Mohammad Alibakhshi Kenari. This is an open access article distributed under the Creative Commons Attribution \\ License, which permits unrestricted use, distribution, and reproduction in any medium, provided the original work is properly cited.
}

\begin{abstract}
Design of new metamaterial (MTM) traveling-wave antenna with "d-formed" left-handed structure is proposed. The proposed MTM structure is designed at a height of $0.8 \mathrm{~mm}$ from the ground plane with almost $2 \mathrm{GHz}$ bandwidth. The most interesting feature of the design is the ability of enhancing the gain and total efficiency of the antenna without negative effects of the other important parameters like bandwidth. By using the "d-shaped" MTM structure and printed planar technique, the bandwidth of the MTM traveling-wave antenna is significantly increased at a resonant frequency of $6 \mathrm{GHz}$ and also a foot print area reduction of the antenna structure is provided. Antenna size is $7.2 \times 5 \times 0.8 \mathrm{~mm}^{3}$. The proposed antenna is suitable for RF portable devices operating at $6 \mathrm{GHz}$.
\end{abstract}

Keywords: Small Broad Band Antenna; Metamaterial (MTM); d-Shaped Antenna Structure; RF Portable Devices; Traveling-Wave Antenna

\section{Introduction}

A printed patch antenna $[1,2]$ is a low-profile antenna consisting of a metal layer over a dielectric substrate and ground plane. Typically, a printed patch antenna is fed by input signal, but other feed lines such as coaxial and microstrip transmission line can be used. The advantages of printed patch antennas are that they radiate with moderately high gain in a direction perpendicular to the substrate and can be fabricated in a low cost printed circuit board (PCB). The basic operating principle of a printed patch antenna is that the space between the patch and ground plane acts like a section of parallel plate waveguide. Neglecting radiation loss, the edge of the patch is an open circuit, so that energy reflects and remains below the patch. One disadvantage of a high-Q system is narrow bandwidth, so patch antennas have limited bandwidth, meaning that the input impedance of the antenna only remains near the desired value for a small range around the designed center frequency. In this paper, to overcome this challenge we used MTM technology, to extend the antenna bandwidth.

MTMs represent a new paradigm in electromagnetic science and technology $[3,4]$. Some of the applications of the MTM antennas are RF units, wireless communication, mobile communication, space communications, global positioning system (GPS), satellites, space vehicle navigation, and airplanes. They have already led to many unprecedented microwave applications, which may be classified in three categories: guided-wave, radiated-wave and refracted-wave applications [5]. MTM antennas configurations may be of two types. The first type consists of conventional radiators (e.g. patches or dipoles) placed above artificial dielectrics [6], where the main benefits of the artificial dielectric MTMs are compactness [7] and slightly enhanced bandwidth in electromagnetically small antennas [8]. These TL MTMs generally exhibit a composite right/left-handed (CRLH) dispersive response [3], or related responses when the line integrates additional lumped elements in its unit cell (higher order TL) [9].

This paper presents an overview on CRLH resonant antennas (RAs) and also a new concept of design of the printed patch traveling-wave antenna resonating at $6 \mathrm{GHz}$ based on CRLH-TLs.

\section{Brief Review of MTM RAs}

A CRLH TL MTM is an artificial TL structure composed of the periodic repetition of a CRLH unit cell, as is shown in Figure 1. Such a TL exhibits in the lossless 
case the dispersion/attenuation relation [3]

$\cos [(\beta-j \alpha) p]=1+\frac{Z Y}{2}=1-\frac{\left(\omega^{2}-\omega_{s e}^{2}\right)\left(\omega^{2}-\omega_{s h}^{2}\right)}{2 \omega^{2} \omega_{R}^{2}}$,

where $\omega_{s e}=\frac{1}{\sqrt{L_{R} C_{L}}}, \omega_{s h}=\frac{1}{\sqrt{L_{L} C_{R}}}$, and $\omega_{R}=\frac{1}{\sqrt{L_{R} C_{R}}}$. Under balanced condition where $\left(\omega_{s e}=\omega_{s h} \equiv \omega_{0}\right)$, the dispersion relation $\omega(\beta)$ can be explicitly derived from inversion of the general relation (1) as

$$
\omega(\beta)=\sqrt{\omega_{0}^{2}+\omega_{R}^{2} \sin \left(\frac{\beta p}{2}\right)}+\omega_{R} \sin \left(\frac{\beta p}{2}\right)
$$

this dispersion curve is plotted in Figure 2.

In the MTM frequency range $\left(p \ll \lambda_{g}\right)$, the artificial CRLH structure behaves as a uniform TL

$\left[\beta(\omega) \rightarrow \frac{\omega}{\omega_{R}}-\frac{\omega_{L}}{\omega}\right.$, where $\left.\omega_{L}=\frac{1}{\sqrt{L_{L} C_{L}}}\right]$, and may therefore be transformed, like any TL, into a TL resonator by using discontinuous (short/open) terminations. A difference with a uniform TL however is that, due to the CRLH pass-band characteristic, only $2 \mathrm{~N}-1$ resonances, where $N$ is the number of unit cells, can fit in the transmission band. These resonances naturally correspond to TL lengths $l=n \lambda_{g} / 2$, and therefore to propagation constants $\beta_{n}=\frac{n \pi}{l}=n \pi /(N p)$, with the CRLH particularity that $n$ can be both positive (RH range) and negative ( $\mathrm{LH}$ range), and even zero (transition frequency).

The Bloch (periodic structure) impedance is given in

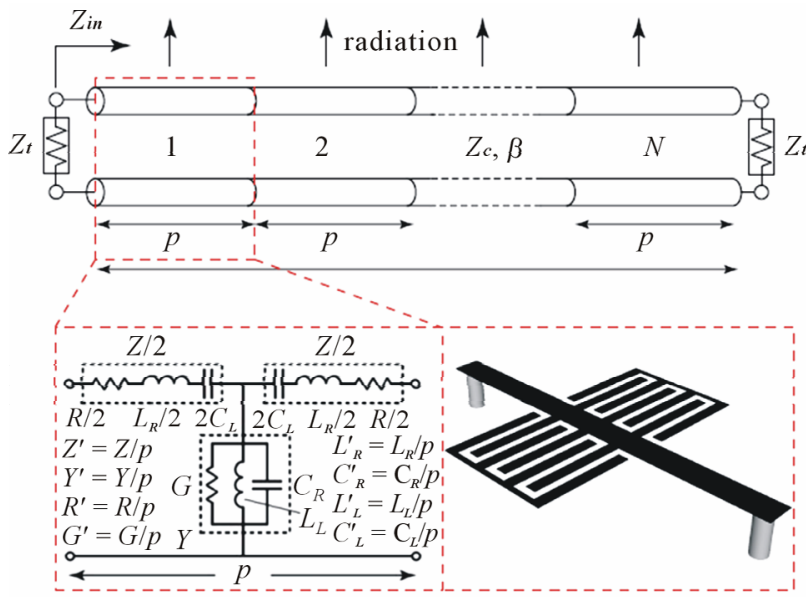

Figure 1. CRLH TL resonant antenna (RA), formed by repeating $N$ times a unit cell of size $p$, where the terminations are either short-ended $\left(Z_{t} \approx 0\right)$ or open ended $\left(Z_{t} \approx \infty\right)$, including unit cell equivalent circuit and typical interdigital/stub implementation. general by $Z_{B}=\sqrt{Z / Y} \sqrt{1+Z Y / 4}$ (as shown in Figure 2). It reduces in the balanced case to $[3,5]$

$$
Z_{B}(\omega)=Z_{L} \sqrt{1+\frac{1}{4}\left[\left(\frac{\omega}{\omega_{R}}-\frac{\omega_{L}}{\omega}\right)^{2}+\delta\right]}
$$

where $Z_{L}=\sqrt{\frac{L_{L}}{C_{L}}}$, and where $\delta=\delta(R, G)$ is a dissipative term, which is much smaller than the reactive terms. In the MTM range, this impedance may be assimilated to a TL characteristic impedance, which is frequency-independent $\left(Z_{B} \rightarrow Z_{C}=Z_{L}=Z_{R}\right.$, where $\left.Z_{R}=\sqrt{\frac{L_{R}}{C_{R}}}\right)$.

In summary, a CRLH resonator supports $2 \mathrm{~N}-1$ resonance frequencies, $\mathrm{N}-1$ in the $\mathrm{LH}$ band, $\mathrm{N}-1$ in the $\mathrm{RH}$ band, and 1 at the transition frequency $\omega_{0}$ (or $\omega_{s e}$ or $\omega_{s h}$, depending on the terminations, if the CRLH structure is unbalanced, $\left.\omega_{s e} \neq \omega_{s h}\right)$. These frequencies are obtained by sampling Equation (1) at the $\beta$ points $\beta_{n}=n \pi / N p,(n=1,2, \cdots 2 N-1)$

$$
\omega_{n}=\sqrt{\omega_{0}^{2}+\omega_{R}^{2} \sin ^{2}\left(\frac{n \pi}{2}\right)}+\omega_{R} \sin \left(\frac{n \pi}{2}\right) .
$$

The Bloch impedance $Z_{B}$ given by Equation (3) is the input impedance when the structure is infinitely periodic or terminated by a resistor of value $Z_{B}$. Since the terminations $Z_{t}$ of the CRLH resonator (Figure 2) are short or open circuits, the impedance at the input of the structure is naturally different from $Z_{B}$.

However, as pointed out in the previous paragraph, $Z_{B}$ constitutes the equivalent TL characteristic impedance $Z_{c}$ in the MTM regime $\left(p / \lambda_{g} \rightarrow 0\right)$, and is therefore essential for coupling energy into (i.e. matching) the resonant structure. The resistive elements $R$ and $G$ in Figure 1 represent in general the radiation resistance in the antenna, in addition to the conductor and dielectric losses, respectively.

As all CRLH MTM structures, CRLH RAs may be implemented in different technologies [planar hybrid or MMIC, LTCC, hollow waveguide], in different waveguide or TL configurations [microstrip, coplanar waveguide (CPW), coplanar strip-line (CPS), coaxial, waveguide, etc.], and using different $L C$ elements [printed or chip form; interdigital (ID) or metal-insulator-metal (MIM) capacitors; straight, spiral, meander inductors]. Some typical implementations are shown in Figure 3. The properties of the resulting antennas-including polarization, radiation patterns, efficiency, directivity-depend not only on the selection of the CRLH resonant mode, but 


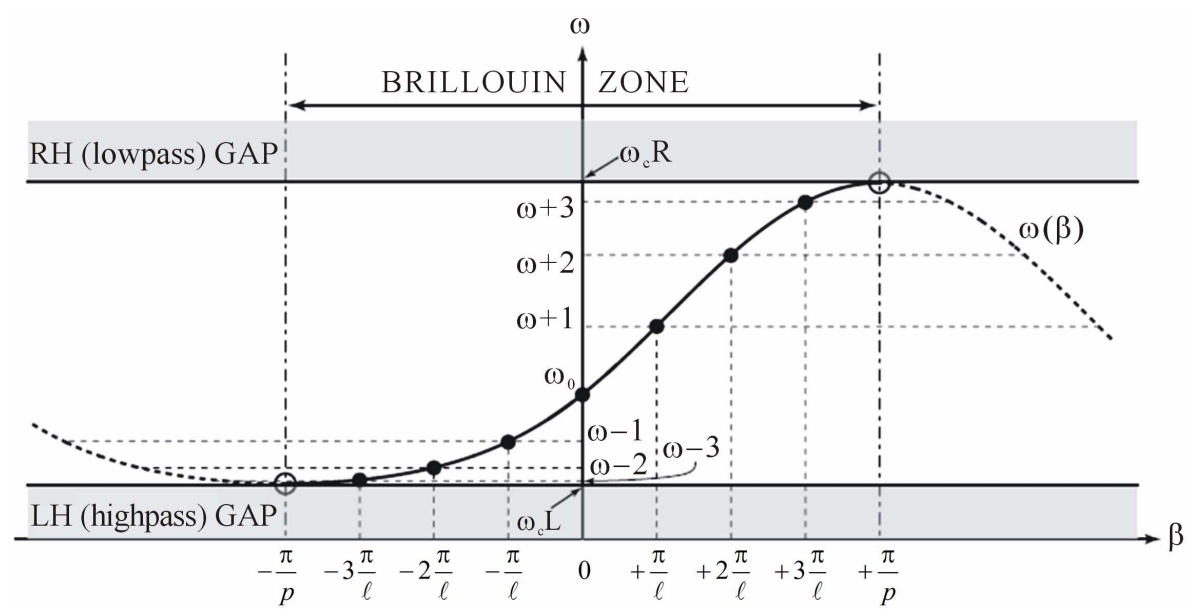

Figure 2. Resonances of the (balanced) CRLH TL resonator shown in Figure 1. The length $l$ and the period $p$ of the resonator are related by $l=N p$, which results in Brillouin zone edges of $\beta_{B Z E}= \pm \frac{\pi}{P}= \pm N \pi / l$.

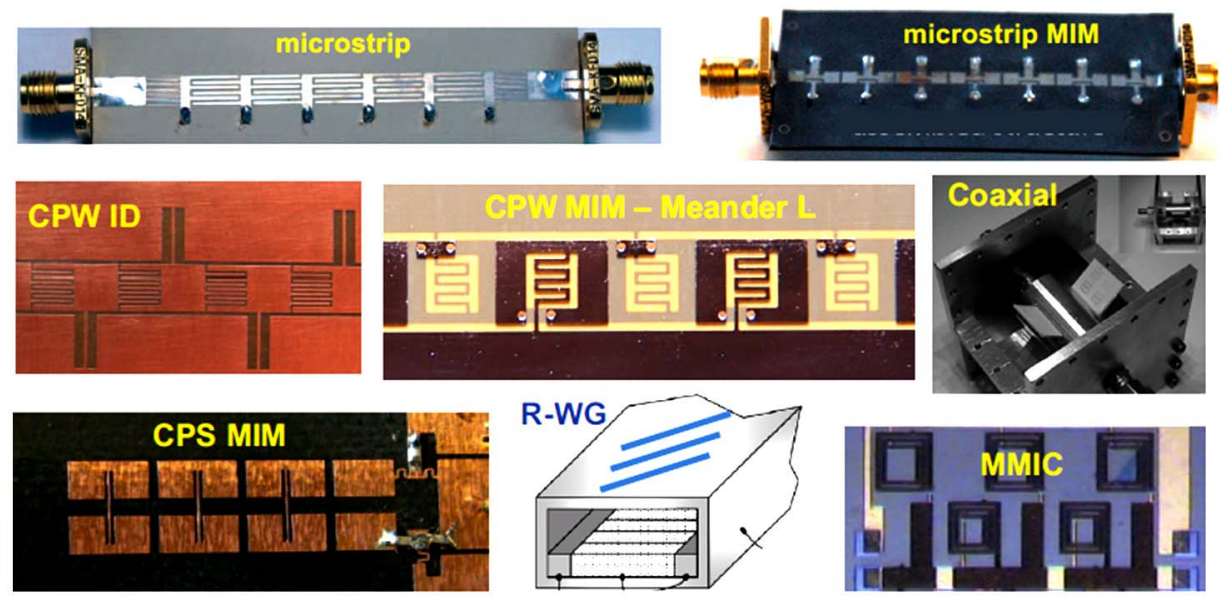

Figure 3. Typical 1D CRLH TL implementations [5].

also on the choice of the technology, configuration, and $L C$ elements.

\section{Design Methodology of Proposed Traveling-Wave Antenna Structure Resonating at $6 \mathrm{GHz}$}

This paper introduces a new and distinct MTM structure named to "printed $\mathbf{d}$-formed antenna" as is shown in Figure 4. The proposed antenna is constructed of two d-shaped unit cells. Each of unit cells consists of d-formed gaps printed on a rectangular radiation patch. These gaps act like series capacitances $\left(C_{L}\right)$. Also, from spiral inductors with two turns that through metallic via holes are connected to the ground plane and perform the roles of shunt inductors $\left(L_{L}\right)$.

The dimension of printed patch antenna is $7.2 \mathrm{~mm} \times 5$ $\mathrm{mm} \times 0.8 \mathrm{~mm}$, which are shown in Figure 4(b). This antenna is designed on FR-4 (Lossy) substrate with Tan $\delta$ $=0.001$, dielectric constant of $\varepsilon_{r}=4.6$ and height from the ground plane of $\mathrm{h}=0.8 \mathrm{~mm}$. Two waveguide ports were defined at the left and right of the x-axis in order to calculate the $S_{11}$ and $S_{21}$ parameters as are shown in Figure 4(a). Port 1 is excited by input signal and port 2 is matched to $20 \mathrm{ohm}$ load impedance of the SMD components which is connected to ground through via hole.

To implement a small antenna, we used MTM technology and printed planar technique, as printed gaps acts like left-handed capacitors $\left(C_{L}\right)$ and leads to foot print area reduction, and also to create broadband performances have used of spiral inductors, so these inductors performs the role of left handed inductors $\left(L_{L}\right)$. In addition, new hypotheses will be discussed in the following referred to it. Overall size of the proposed antenna is 7.2 $\times 5 \times 0.8 \mathrm{~mm}^{3}$ or $0.11 \lambda_{0} \times 0.079 \lambda_{0} \times 0.012 \lambda_{0}$, where $\lambda_{0}$ is free space wavelength at $4.7 \mathrm{GHz}$. 


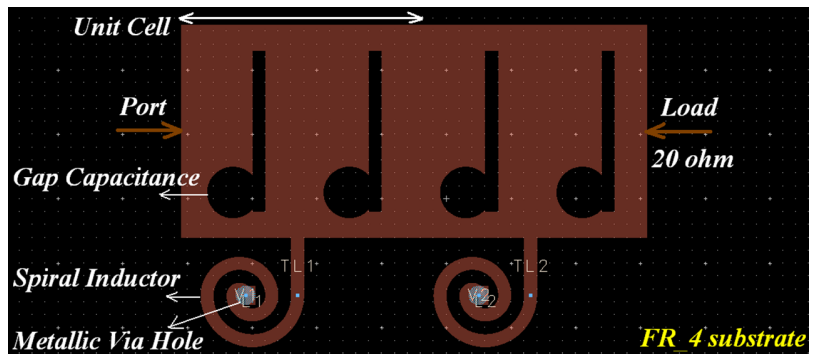

(a)

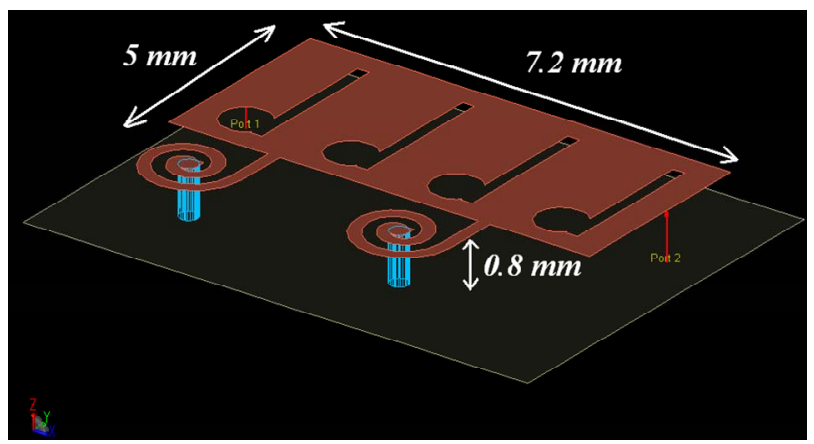

(b)

Figure 4. Configuration of the proposed traveling wave antenna constructed of two unit cells. a) layout of the top view, b) layout of the isometric view.
This antenna covers frequency bandwidth from 4.7 to $6.7 \mathrm{GHz}$ and from 4.9 to $6.6 \mathrm{GHz}$ that were derived from ADS and HFSS softwares, respectively. Equivalent circuit model of the d-formed antenna is displayed in Figure 5.

Shunt capacitance $\left(C_{R}\right)$ and series inductance $\left(L_{R}\right)$ are right-handed parasitic effects which unavoidably are created due to existence of spaces between patches and ground plane and current flows on the patches. Losses of the structure are modeled by $R_{R}, G_{R}, R_{L}$ and $G_{L} . R_{R}$ and $G_{R}$ are right-handed losses and $R_{L}$ and $G_{L}$ are left-handed losses.

In this paper, for realization of the small wideband antenna [10], we employed new ideas based on MTM technology of printed planar patches and smaller value of the loaded series capacitance. For implementation of the small dimensions, $\mathbf{d}$-shaped copper artwork is printed directly on a printed circuit board using standard printed circuit board manufacturing techniques, so leads to output series capacitor $\left(C_{L}\right)$, thus can be leading to a reduction in antenna dimensions. In addition, for implementing a smaller value of the loaded series capacitance, we utilized the least of spaces that are printed on radiation patches which provides series capacitances and leading to reduce of the printed area and its effect bandwidth of

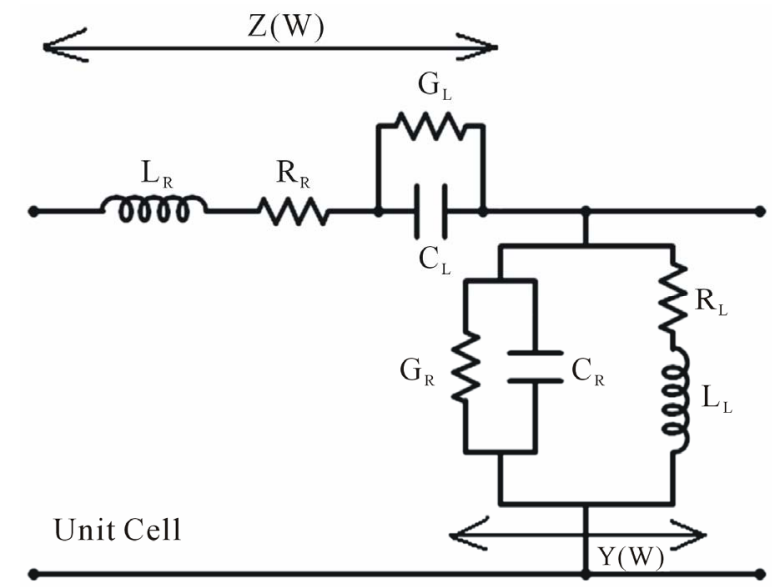

(a)

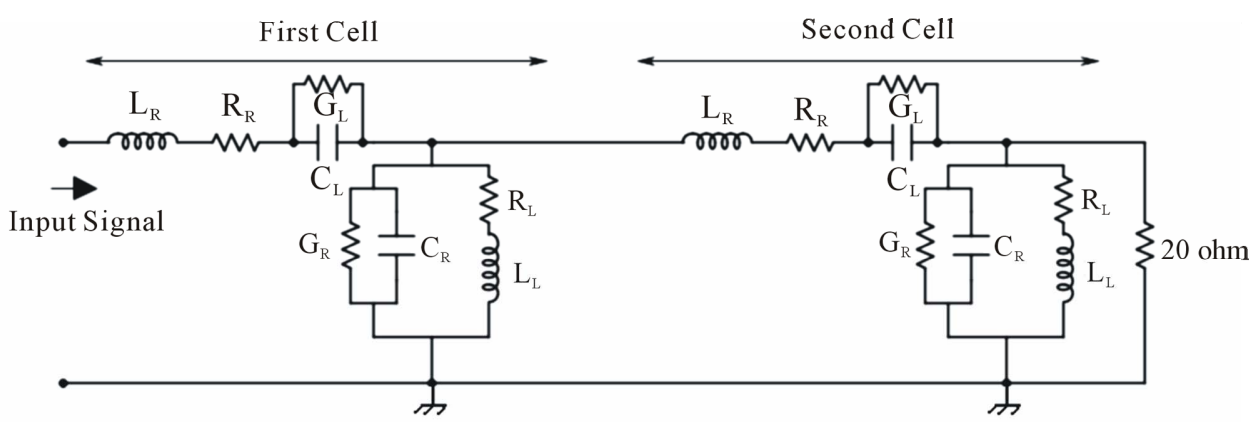

(b)

Figure 5. Equivalent circuit model of the minimized traveling wave antenna. a) unit cell, b) entire structure. 
the antenna has been extension. The length, width and height of proposed antenna are $7.2 \mathrm{~mm}, 5 \mathrm{~mm}$ and 0.8 $\mathrm{mm}$ and the structure can be used for an impedance bandwidth $\left(S_{11}<-10 \mathrm{~dB}\right)$ from 4.7 to $6.7 \mathrm{GHz}$ that is obtained by using ADS simulator and from 4.9 to 6.6 $\mathrm{GHz}$ that was obtained by using HFSS simulator, that are equal to 2 and $1.7 \mathrm{GHz}$ bandwidth which corresponds to $35.08 \%$ and $29.56 \%$ practical bandwidth, respectively. The reflection coefficients $\left(S_{11}<-10 \mathrm{~dB}\right)$ of the proposed traveling-wave antenna are exhibited in Figure 6.

Other important issue in design of the antenna systems is radiation pattern. Therefore, acceptable radiation characteristics are necessary properties of the antenna systems. In this framework, beside small size and wide bandwidth characteristics, we have suggested a new idea for providing good radiation performances. For obtaining suitable radiation properties, we have employed of the uniform excitation mechanism by utilizing two ports, as port 1 is excited by input signal and port 2 is matched to $20 \mathrm{ohm}$ load impedance. Furthermore, in design procedure of the proposed antenna for obtaining appropriate radiation performances is used of inductive and capacitive elements with optimized values, so that lead to expansion of the aperture efficiency, therefore radiation characteristics have been increased. Maximum radiation gains and efficiencies of the proposed small and wideband antenna at $6 \mathrm{GHz}$, derived from ADS and HFSS simulators, are $3.6 \mathrm{dBi}$ and $60.3 \%$, and $3.3 \mathrm{dBi}$ and $48.2 \%$, respectively. The radiation gains patterns of the antenna at $6 \mathrm{GHz}$ are plotted in Figure 7. It is clear that, radiation patterns of the proposed antenna have unidirectional characteristics.

Therefore, suggested antenna is include advantages of small size, in other hand, has dimension of $7.2 \times 5 \times 0.8$ $\mathrm{mm}^{3}$ or $0.11 \lambda_{0} \times 0.079 \lambda_{0} \times 0.012 \lambda_{0}$, wideband performances with about $2 \mathrm{GHz}$ bandwidth from almost 4.7 to almost $6.7 \mathrm{GHz}$, which are related to approximately

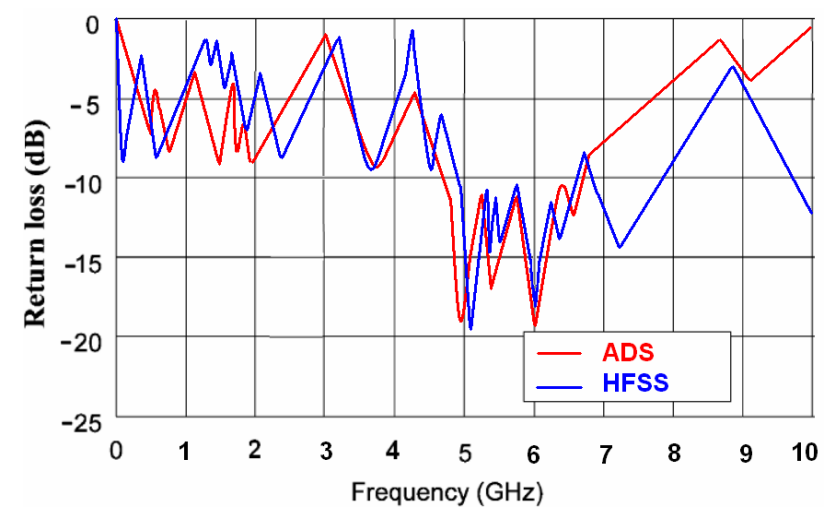

Figure 6. Return losses $\left(S_{11}<-10 \mathrm{~dB}\right)$ of the proposed d-shaped antenna which were obtained from ADS and HFSS simulators.

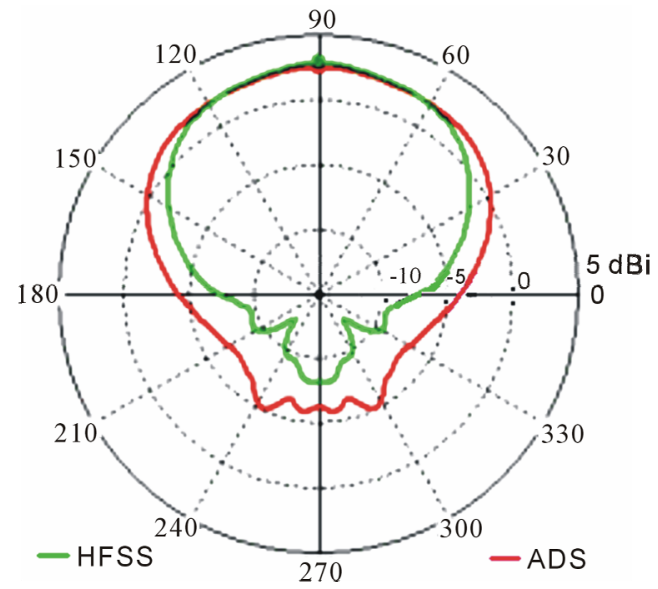

Figure 7. Radiation gains patterns of the traveling-wave antenna in elevation plane $\left(\Phi=0^{\circ}\right)$ at $6 \mathrm{GHz}$, which were derived from ADS and HFSS simulators.

$35.08 \%$ practical bandwidth and excellent radiation properties with maximum gain of $3.6 \mathrm{dBi}$ and radiation efficiency of $60.3 \%$ that were obtained from ADS simulator, so that highest these parameters derived from HFSS simulator are equal to $3.3 \mathrm{dBi}$ and $48.2 \%$, respectively, that occurs at resonating frequency $\mathrm{f}=6 \mathrm{GHz}$.

It is noteworthy that, increase the number of unit cells will lead to the relative increase in gain, but in return, it will adversely affects on the size and bandwidth of the antenna, so that leads to somewhat lower bandwidth and increase the size.

To validate the design, the simulation processes of the antenna are done using Advance Design System (ADS) and High Frequency Structure Simulator (HFSS) simulators.

According to obtained results the proposed travelingwave antenna resonating at $6 \mathrm{GHz}$ based on CRLH MTMTLs can be appropriate nominee for wireless applications and RF portable devices.

\section{Conclusions}

In this paper, we presented new concepts of design of the small wideband traveling-wave antenna resonating at $6 \mathrm{GHz}$ based on MTM technology, printed patch technique, smaller value of the loaded series capacitance and use of suitable inductive and capacitive components with their optimized amounts.

The "d-shaped" MTM structure with rectangular patch antenna is proposed. Overall size of this antenna structure is very compact in comparison to conventional antennas. On making $\mathbf{d}$-shaped structure by double negative left-handed MTMs, antenna parameters such as dimension, gain, efficiency, and bandwidth can be improved up to a desired limit but practical limitations should be taken care of while fabricating the structure with ADS and 
HFSS softwares. The proposed antenna has benefits of low cost, ease of implementation, unidirectional radiation patterns, high gain and efficiency, broader bandwidth than conventional wideband antennas and smaller size than conventional compact antennas and this antenna can be fabricated in a low cost and low-profile printed circuit board (PCB) using standard PCB manufacturing techniques.

\section{Acknowledgements}

The author would like to express his sincere thanks to Iran Telecommunication Research Center, i.e.; ITRC (Contract number 6987/500/T), the microwave and millimeter wave laboratory of the Amirkabir University of Technology (Tehran Polytechnic) and the antenna laboratory of the K. N. Toosi University of Technology.

\section{Author Biography}

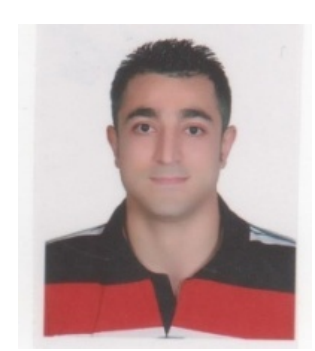

Mohammad Alibakhshi Kenari was born in 1988 at Iran, Mazandaran, Freydunkenar. He received the B.S. and M.S. degrees in communication engineering from the university of Najafabad at Iran, in 2009 and the university of Shahid Bahonar at Iran in 2013, respectively.

His research involves microwave and millimeter wave circuits, minimized and wideband antennas, MTM CRLH-TLs implementations, FR portable devices and embedded systems.

\section{REFERENCES}

[1] C. A. Balanis, "Antenna Theory and Design,” John Wiley \& Sons, Hoboken, 1997.
[2] S. N. Burokur, Mo. Latrach and S. Toutain, "Theoritical Investigation of a Circular Patch Antenna in the Presence of a Left-Handed Mematerial," IEEE Antennas and Wireless Propagation Letters, Vol. 4, 2005, pp. 183-186.

[3] C. Caloz and T. Itoh, "Electromagnetic Metamaterials, Transmission Line Theory and Microwave Applications," Wiley and IEEE Press, Hoboken, 2005. doi: $10.1002 / 0471754323$

[4] N. Engheta and R. W. Ziolkowski, "Electromagnetic Metamaterials: Physics and Engineering Explorations," Wiley and IEEE Press, Hoboken, 2006.

[5] C. Caloz, T. Itoh and A. Rennings, "CRLH TravelingWave and Resonant Metamaterial Antennas," Antennas and Propagation Magazine, Vol. 50, No. 5, 2008, pp. 25-39. doi:10.1109/MAP.2008.4674709

[6] R. E. Collin, "Field Theory of Guided Waves," 2nd Edition, Wiley-Interscience, 1991, Chapter 12.

[7] M. Coulombe, H. V. Nguyen and C. Caloz, "Substrate Integrated Artificial Dielectric (SIAD) Structure for Miniaturized Microstrip Circuits," Antennas and Wireless Propagation Letters, Vol. 6, No. 6, 2007, pp. 575-579. doi:10.1109/LAWP.2007.910959

[8] P. M. T. Ikonen, K. N. Rozanov, A. V. Osipov, P. Alitalo, and S. A. Tretyakov, "Magnetodielectric Substrates in Antenna Miniaturization: Potential and Limitations," Transactions on Antennas and Propagation, Vol. 54, No. 6, 2006, pp. 3391-3396. doi:10.1109/TAP.2006.884303

[9] A. Rennings, S. Otto, J. Mosig, C. Caloz and I. Wolff, "Extended Composite Right/Left-Handed (E-CRLH) Metamaterial and Its Application as a Quadband Quarter Wavelength Transmission Line," Asia-Pacific Microwave Conference, (APMC), Yokohama, 12-15 December 2006, pp. 1405-1408.

[10] Anon, "FCC First Report and Order on Ultra Wideband Technology," 2002. 